\title{
A Physical Model of the Interphase Boundary of the Passivating Layer on the Metal in the Electrolyte Solutions
}

\author{
SAIF A. MOUHAMMAD* and AYMAN F. AMER \\ Department of Physics, Taif University, Saudi Arabia. \\ ${ }^{*}$ Corresponding author E-mail: saifnet70@ hotmail.com \\ http://dx.doi.org/10.13005/ojc/300235 \\ (Received: March 01, 2014; Accepted: March 27, 2014)

\section{ABSTRACT}

\begin{abstract}
The model of processes on the interphase boundary metal- passivating layer is proposed. The balance of ions upon the formation of the cells of oxide is examined. The velocity expressions of these processes are obtained.
\end{abstract}

Key words: Physical model, Passivity of metals, Electrolyte.

\section{INTRODUCTION}

The mechanism of processes on the interphase boundary of the passivating oxide layer $\mathrm{PL}$ has a fundamental value, whereas, the properties of the passivating layer are determined by them.In this paper a consistent model of the boundary metal-oxide MO is presented.

\section{General stationary laws.}

On the interphase boundary of metal with $\mathrm{PL}$, the continuous dissolutionof metal occurs.it disappears consecutively monolayer after a monolayer and the cells of oxide occupy its place. if at time $\Delta \mathrm{t}$ the layer of metal $\Delta \mathrm{M}$ of thickness $\Delta \mathrm{x}$ disappears, then the plane $\mathrm{MO}$ displaces in its side with the speed $V$, equals

$$
v=\Delta x / \Delta t=\left(i_{b} / z F\right)(\mu / \rho)
$$

where $\mathrm{i}_{b}$ - total dissolution current; $\mu, \rho$ - the molar mass and density. The thickness of the oxide layer $\Delta_{\mathrm{ok}}$, substitutingfor the same time, is also equal.

let us designate a quantity of ions in the initial layer $\Delta \mathrm{M}$ of metal and its substituted layer of oxide $\Delta$ oK by $\Delta N$ and $\Delta N_{1}$ respectively. It is obvious, that $\Delta \mathrm{N}_{1}<\Delta \mathrm{N}$, since the specific volumes, according to the metal ion, in $\Delta \mathrm{M}$ and $\Delta_{\mathrm{ok}}$ are different. Consequently, only ions $\Delta \mathrm{N}_{1}$ in a layer $\Delta \mathrm{M}$ involved in the formation of oxide $\Delta_{\mathrm{ok}}$. The remaining part, equal $\Delta \mathrm{N}_{2}=\Delta \mathrm{N}-\Delta \mathrm{N}_{1}$, considered to be excess and must leave into the solution through the boundary $\mathrm{MO}$ and PL. Therefore, the balance of space in the layer of metal $\Delta \mathrm{M}$ and the oxide $\Delta_{\mathrm{ok}}$ is observed. 
Thus, the surface $\mathrm{MO}$ serves as the source of ions $\mathrm{M}^{z+}{ }_{0 k}$, and in the passivating layer, their intensive transfer by the mechanism of hopping migration in the strong electric field $E$ should be occurred. Accordingly, the total current of the dissolution of metal is divided into the transfer current $\mathrm{i}_{\mathrm{bm}}$ of the ions through the boundary $\mathrm{MO}$ in $P L$ and the current $\mathrm{i}_{\mathrm{bo}}$ of oxide formation on this boundary (subscripts indicate: b- boundary, mmetal, o- oxygen; the current $i_{b 0}$ determines a quantity of metal ions, which in $1 \mathrm{~cm}^{3}$ every second are connected in the layer $\Delta \mathrm{M}$ with oxygen), such as

$$
\begin{aligned}
& \Delta N_{1}+\Delta N_{2}=\Delta N, \\
& i_{b m}+i_{b o}=i_{b} .
\end{aligned}
$$

The subsequent analysis confirms this conclusion. The total current must be equal

$$
\begin{aligned}
& i_{b}=i_{b 0} \exp \left(a_{m} \beta E\right), \quad \beta=\alpha_{b} z_{m} F / R T, \\
& E=\left(\varphi-\varphi_{b}\right) / \delta(\varphi), \quad \varphi_{m}=a_{m} E,
\end{aligned}
$$

Where $a_{m}$ - interatomic distance in the cationic sublattice, $a_{b}$ - transfer coefficient on the boundary MO.

Equation(3) assumes, that an ionM, passing into the state of anion $M_{s k}^{*+}$, must overcome a potential jump $\varphi_{M}=a_{M} E$. Thus, the barrier of transition decreases to $\Delta \mathrm{W}_{\mathrm{M}}=\mathrm{a}_{\mathrm{b}} \mathrm{Z}_{\mathrm{M}} \varphi_{\mathrm{M}}$. The constant of transition speed is determined from the independent temperature measurements ( for $\mathrm{Fe}$, for example, ).

The total current $i_{b}$ is the fundamental characteristic of stationary processes on the interphase boundary $\mathrm{MO}$, and the micro scheme of the formation of cells $\mathrm{M}_{2} \mathrm{O}_{3}$ are ambiguous. They must consider a difference between the interatomic distances and the angular directions in the lattices of metal and oxide, and require the use of probabilistic methods for describing regrouping particles.

Further, it is interesting to detail a physical situation on a surface $\mathrm{MO}$ to calculate the currents $\mathrm{i}_{\mathrm{bm}}, \mathrm{i}_{\mathrm{bo}}$ and the ratio between the values $\Delta \mathrm{N}_{1}$ and $\Delta \mathrm{N}_{2}$. For this purpose let us proceed from general consideration to the specific models of processes on the boundary MO.The most simple and real vacancy model is presented below. It allows to open a better physical sense of therelations (3).

\section{The vacancy model of MO boundary}

Vacancy model proceeds from the idea about the fact that, for forming the new cell $\mathrm{M}_{2} \mathrm{O}_{3}$, three ions $\mathrm{O}^{2-}{ }_{\text {ok }}$ from the passivating layer must penetrate in the boundary monolayer of metal. This requires the presence of three vacancies $V_{M}$ in it, which can arise only as a result of previous transition in the passivating layer of three ions of metal. These ions fill three vacancies $V_{\text {om }}$ in the cation'ssublattice of oxide. The transition scheme $\left(\mathrm{V}_{\mathrm{M}}\right.$ - vacancy in a metallicsublattice of oxide,- vacancy in the boundary layer of the metal $\Delta \mathrm{M}$ )

$$
M+V_{o m} \rightarrow M_{o k}^{z+}+V_{m}+z e
$$

Transition (4) serves as the necessary condition for the subsequent penetration into the layer of oxygen ions

$$
\mathrm{O}_{o k}^{2-}+M+V_{m} \rightarrow \mathrm{MO}_{z / 2}+V_{o k}+z e
$$

Where $\mathrm{V}_{\mathrm{ok}}{ }^{-}$vacancy in the oxygen sublattice in place passedinto the metal ion $\mathrm{O}^{2-}{ }_{\text {ok }}$.

The opposite reactions (4), (5)- appear basic in the vacancy model. It is obvious, that the surface MO serves as a drainof cationic vacancy $\mathrm{V}_{\mathrm{om}}$, brought by their diffusive flow $\mathrm{j}_{\mathrm{mv}}$ from solution. Simultaneously, it is the source of the vacancies ${ }_{\text {om }}$, which are discharged into the solution by their flow $\mathrm{j}_{\mathrm{ov}}$. Flows $\mathrm{j}_{\mathrm{mv}}$ and $\mathrm{j}_{\mathrm{ov}}$ appear, of course, as a result of the motion of ions $\mathrm{M}^{2+}{ }_{\text {ok }}$ and $\mathrm{O}^{2-}{ }_{\text {ok }}$ in the passivating layer by the mechanism of hopping migration in the strong field.

The transformation of vacancies and their flows occurs on the surface MO by the scheme.

$$
\begin{aligned}
& V_{o m} \rightarrow V_{m} \rightarrow V_{o k} \\
& j_{m v} \rightarrow j_{o v}
\end{aligned}
$$


in this transformation, the vacancies $\mathrm{V}_{\mathrm{m}}$ are mediators, which connect processes (4) and (5). Their total quantity is determined by the withdrawal of metal ions and penetrationin metal of the oxygen ions $\mathrm{O}^{2-}{ }_{\text {ok }}$, i.e. the relations of the speeds (4) and (5).In the stationary mode, the value of is invariable ( see below).

The speeds of formation of oxide and cation transfer through the boundarymo.

Thus, processes On the boundary $\mathrm{MO}$ are characterized by the currents (speeds) $\mathrm{i}_{\mathrm{bm}}, \mathrm{i}_{\mathrm{bo}}$ of reactions (4), (5) $i_{b m}, i_{b o}$ and by the total current $i_{b}$ of the metal dissolution. It is measured, and is primary value, and its determination (3) can be called extramodel. The calculation of currents $\mathrm{i}_{\mathrm{bm}}, \mathrm{i}_{\mathrm{bo}}$ depends on model presentations. let us examine these values.

1) Transition current $i_{b m}$ of ions $M$ in the passivating layer andtheir further transfer to the solution depends on the electric field $E$ and the boundary concentration $\mathrm{a}_{\mathrm{m}} \mathrm{N}_{\mathrm{mv} 2}$ of vacancies $\mathrm{V}_{\mathrm{om}}$. Indeed, for transition into the passivating layer according to the scheme (4), ions M must cross the plane $\mathrm{MO}$ and move away from it on interatomic distance $a_{m}$ of a cationic sublattice, having overcome potential jump $\varphi_{\mathrm{m}}=\alpha_{\mathrm{m}} \mathrm{E}$. Transition barrier is reduced on $\Delta W_{m}=\alpha_{b} z_{m} a_{m} E$, its probability is proportional to a quantity of vacant places, i.e., the concentration $a_{m} N_{m v 2}$ of vacancies $V_{m}$. As a whole the speed (4) is equal

$$
\begin{cases}i_{b m}=i_{b m}^{(0)} \exp \left(\beta a_{m} E\right), & \beta=\alpha_{b} z_{m} F / R T, \\ i_{b m}^{(0)}=\bar{i}_{b m} a_{m} N_{m \cdot 2} N_{m e t}, & \bar{i}_{b m} \sim \exp \left(-W_{b} / K T\right), \\ E=\left(\varphi-\varphi_{p}\right) / \delta(\varphi), & \varphi_{m}=a_{m} E,\end{cases}
$$

$\alpha_{b}$ - transfer coefficient , $\mathrm{Nm}_{\mathrm{et}}$ - concentration of atoms in metal, - $\bar{i}_{b m}$ speed constant of reaction.

2) The current $i_{b o}$ of ions $M$, which form oxide $\mathrm{MO}_{\mathrm{z} / 2}$ according to the reaction (5), represents the speed of oxide formation on the Boundary MO.let us take it equal to $\left(\bar{i}_{b m}\right.$ - constant of speed, reverse current is neglected)

$$
\begin{aligned}
& \int i_{b o}=i_{b o}^{(0)} c_{m e}^{\not / z}\left(N_{a 2} / N_{a 0}\right) f(u) \exp \left(\bar{\beta} a_{a} E\right), \\
& \bar{\beta}=\bar{\alpha}_{b} z_{a} F / R T, \quad i_{b o}^{(0)}=i_{b o} N_{a 02} a_{m}^{2 / t}, \\
& c=N_{v s} / N_{s}, \varphi_{o}=a_{o} E, \quad N_{s 2}=N_{s 0}-N_{s v}
\end{aligned}
$$

In the system (7), the dependence of current $\mathrm{i}_{\mathrm{bo}}$ on the field $\mathrm{E}$, the concentration $\mathrm{c}_{\text {met }}$, the vacancies $V_{m}$ and the probabilistic factor $f(u)$ are taken into account. the influence of field is regulated by the value of transfer coefficient $\bar{a}_{2}$, and it is connected with the fact, that for the penetration into the metal through the plane $\mathrm{MO}$, ion $\mathrm{O}^{2-}{ }_{\text {ok }}$ must dislocate in the anion sublattice to the interatomic distance $a_{o}$, overcoming the potential jump $\varphi_{0}=a_{0} E . I n$ this case, the barrier of penetration is reduced to the value $\Delta W_{0}=a_{b} z_{o} a_{0} Z$. Influence on current $\mathrm{i}_{\text {bo }}$ of concentration $\mathrm{c}_{\text {met }}$ is considered with the fact, that without vacancies $V_{m}$, penetration can't occur (see (5)).

The Probabilityfactor $f(u)$ in (7) takes into account the specific mechanism of formation of cells $\mathrm{MO}_{\mathrm{z} / 2}$. If the penetration of ions and the structuring $\varphi(f)$ cells occur in parallel, then it is not possible to exclude the deceleration necessary of regrouping of particles. The micro schemes of these processes can be different and random. Their possible influence on the speed of formation of oxide is described by probabilistic factor $f(u)$ in (7). Its calculation is an autonomous task.

If the penetration of ions $\mathrm{O}^{2-}$ ik is limited and it is weakly dependent on the subsequent structuring, then . Within the limit of the passivating layer it can consist of the partially structure systems complexes $\mathrm{M}_{2} \mathrm{O}_{3}$, the speed of formation of which is determined by $(7)$ at $f(u)=1$. Structuring depends, in particular, on the noncoincidence of interatomic distances and angular orientations in the lattices of metal and oxide.

Transfer coefficients $a_{b}$ and $\beta_{b}=b 1-\alpha_{b}$ in (6) and (7) characterize asymmetry barrier $\mathrm{W}$, which overcome by ions during their motion on the units of crystal lattice in the direct and opposite directions. Depending on $\alpha$ and $\beta$, how much an electric field changes barrier $W$. In the case of an isotropic 
gomogennmedium $\alpha_{b} \sim \beta_{B} \sim \frac{1}{2}$. However, in a thin passivating layer on the strange base layer, these values $\alpha_{b}$ and $\beta_{b}$ are not proved. Furthermore, the current values $\alpha_{b}, \beta_{b}$ in the volume of the passivating layer, and its more disordered boundaries may differ, i.e they depend on coordinate system. Apparently, in this respect, the boundary $\mathrm{MO}$ is isolated, on which exactly $\alpha=\alpha_{b}, \beta=\beta_{\mathrm{B}}$. Then the jumps of the potential $\varphi_{\mathrm{m}}$ and $\varphi_{\mathrm{o}}$ overcome by ions $\mathrm{M}$ and $\mathrm{O}^{2-}{ }_{\text {ok }}$ upon transition through the plane $\mathrm{MO}$ are equal respectively to $\varphi_{\mathrm{m}}=\mathrm{a}_{\mathrm{m}} \mathrm{E}$ and $\varphi_{\mathrm{m}}=\mathrm{a}_{\mathrm{o}} \mathrm{E}$.

Barriers(4) in the Boundary $\mathrm{MO}$ are reduced on $\Delta W_{m}=a_{b} z_{m} \varphi_{m}$ and. All this are taken into account in (6) and (7).

Using (6), (7) it is possible to calculate stationary concentration of vacancies. Actually, the withdrawal of ions $\mathrm{M}$ and the penetration of ions $\mathrm{O}^{2-}$ ${ }_{\text {ok }}$ are strictly compensated (for example, for formation $\mathrm{M}_{2} \mathrm{O}_{3}$, three ions $\mathrm{M}$ have to leave metal and three ions $\mathrm{O}^{2-}{ }_{\mathrm{ok}}$ will penetrate in it).

for example, for forming, three ions must leave from the metal and three ions will penetrate in it. Therefore, speeds (6) and (7) are identical, i. e. $\frac{2}{3} i_{b m}=i_{b o}$ and, consequently (excluding small reverse currents)

$$
\begin{aligned}
& c_{\text {met }}=\frac{2}{3} \frac{i_{b m}^{(0)}}{i_{b 0}^{(0)}} \frac{\exp \left\{E\left(\beta a_{m}-\beta a_{k}\right)\right\}}{\left(N_{o 2} / N_{o 0}\right) f(u)}, \\
& N_{o 2}=N_{o 0}-N_{v o}
\end{aligned}
$$

3) total current in $\mathrm{i}_{\mathrm{b}}$ the vacancy model is easy to relate with the values $i_{b m}$ and $i_{b 0}$ taking into account, that the dissolution of the metal at the interface MO is fasteroxide formation. Actually, according to the presented above, for the appearance of a cell $\mathrm{M}_{2} \mathrm{O}_{3}$ it is necessary for emergence of a cell, that: a) three ions of metal from a layer $\Delta \mathrm{M}$ passed to $\mathrm{PL}$ by the scheme (4), composing the current $i_{b m}$; b) three ions from a passivating layer enetrated into a layer according to the scheme (5); c) since, the cell $\mathrm{M}_{2} \mathrm{O}_{3}$ contains two ions $\mathrm{M}^{2+}{ }_{\text {ok }}$, then two ions of metal in the $\Delta \mathrm{M}$ layer must be connected with three penetrating ions of oxygen $\mathrm{O}^{2-}{ }_{\mathrm{ok}}$ respectively, to form a complex $\mathrm{M}_{2} \mathrm{O}_{3}$ and to compose the current .

Thus, with the formation of each cell $\mathrm{M}_{2} \mathrm{O}_{3}$, layer loses not two, but five ions of metal, from which three prove to be excess. Their departure from the layer $\Delta \mathrm{M}$ implements balance of space, compensating for the difference in specific volumes for each metal ion in the lattice of a metal oxide. Therefore in the passivating layer the intensive transfer of cations $\mathrm{M}^{\mathrm{z+}}{ }_{\mathrm{ok}}$ by the mechanism of hopping migration occurs. The surface $\mathrm{MO}$ is their intensive source, concentration of $\mathrm{N}_{\text {mv2 }}$ vacancies $V_{\text {om }}$ in (6) is small, the relation of stationary values of currents $m$ and $i_{b o}$ equal $3 / 2$.

The total current is equal to the sum $i_{b}=i_{b m}+i_{b s}=\frac{5}{2} i_{b s}=\frac{5}{2} i_{b m}$ or, accordingly (6),(3)

$$
\begin{aligned}
& i_{b}=i_{b 0} \exp \left(\beta a_{m} E\right), \beta=\alpha_{b} z_{m} F / R T^{\prime} \\
& i_{b 0}=\frac{5}{2} i_{b m}^{(0)}=\frac{5}{2} \bar{i}_{b m} a_{m} N_{m v 2} N_{m e t^{*}}
\end{aligned}
$$

The current of the formation of oxide is equal $i_{b o}=\frac{2}{5} i_{b}$. Every second on $1 \mathrm{~cm}^{2}$ of the plane $\mathrm{MO}$ there is a number of cellsoccurs, which at $\mathrm{z}_{\mathrm{m}}=3$ be equal

$$
n=\frac{i_{b o}}{2 z_{m} e}=\frac{1}{5} \frac{i_{b}}{z_{m} e} .
$$

If the stoichiometry of oxide is different than the previous one, then it will change numerical coefficients [2]. In the general case for the cell $\mathrm{M}_{A} \mathrm{O}_{B}$ we have

$$
\left\{\begin{array}{l}
i_{b o}=\frac{A}{A+B} i_{b}, n=-\frac{1}{A+B}\left(\frac{i_{b}}{e z_{m}}\right), \\
i_{b 0}=\frac{A+B}{B} i_{b m}^{(0)}
\end{array}\right.
$$

With the stated of thevacancy model boundaries $\mathrm{MO}$, self processes at the interface with the opposite solution, considered in the following publications. 


\section{REFERENCES}

1. Vetter, K. Z. Electrochemist. 1951,55, 274; 2. Popov, Y.A. Electrochemist.1985,21, 1496; 1954,58, 230-232 1986, 22, 762-764. 